\title{
Modifiable Risk Factors for Homebound Progression Among Those With and Without Dementia in a Longitudinal Survey of Community-Dwelling Older Adults
}

\author{
Mia Yang ( $\square$ miyang@wakehealth.edu ) \\ Wake Forest Baptist Medical Center \\ Nicholas Pajewski \\ Wake Forest Baptist Medical Center \\ Mark Espeland \\ Wake Forest Baptist Medical Center \\ Douglas Easterling \\ Wake Forest Baptist Medical Center \\ Jeff D. Williamson \\ Wake Forest Baptist Medical Center
}

\section{Research Article}

Keywords: risk factors, homebound progression, dementia, mobility, depression, pain

Posted Date: March 4th, 2021

DOI: https://doi.org/10.21203/rs.3.rs-275313/v1

License: @ (i) This work is licensed under a Creative Commons Attribution 4.0 International License. Read Full License 


\section{Abstract}

OBJECTIVE: Being homebound is independently associated with increased mortality but the homebound population is heterogeneous. In order to improve precision medicine, we analyzed potentially modifiable factors that contribute to homebound progression (from independent to needing assistance, to homebound), stratified by dementia status.

METHODS: Using National Aging and Trends Survey (NHATS), a nationally-representative, longitudinal annual survey from 2011 to 2017 ( $n=11,528$ ), we categorized homebound progression if one transitioned from independent or needing assistance to homebound, including competing risks of institutionalization or death between 2011 and last year of data available for each unique respondent. Using proportional hazards regression, we calculated hazard ratios of potentially modifiable risk factors on homebound progression.

RESULTS: Depression, mobility impairment, and pain increased risk of homebound progression regardless of dementia status. Social isolation increased risk of homebound progression only among those without dementia at baseline.

CONCLUSION: Future care and research should focus on the treatment of depression, mobility, and pain to potentially delay progression to homebound status.

\section{Background}

There are estimated 2 million Americans who are homebound, based on the National Health and Aging Trends Study (NHATS), a cross-sectional, nationally representative sample of community-dwelling, non-institutionalized Medicare beneficiaries ages 65 or older(1). Cognitive and/or physical functional limitations contribute to the homebound continuum, ranging from independence, needing assistance to leave the home, to rarely/never leaving the home (homebound)(1). Medicare's definition of homebound, associated with eligibility to receive home health services, means one requires physical or personal assistance to leave the home and that it requires a "taxing effort"(2), which aligns with both assisted and homebound categories within NHATS(1). Homebound status in older adults indicates vulnerability to worse healthcare outcomes: it is independently associated with more than twice the risk of death, in addition to more comorbidities, more functional impairment, and dementia(1) ${ }^{-}(3)$.

The prevalence of Alzheimer's disease and related dementias (ADRD) is particularly high in older adults who are homebound. ADRD prevalence is strongly correlated with degree of homebound status: $80 \%$ of those who were homebound had dementia, versus $57 \%$ of those who needed assistance and $14.8 \%$ of those who were independent(1).

In 2018, ADRD cost $\$ 277$ billion in the US, and this could rise to $\$ 1.1$ trillion by 2050 , not including care provided by unpaid caregivers, valued at over $\$ 232$ billion(4). Those with dementia have three times the healthcare cost compared to those without dementia, largely due to increased hospitalizations(5) in this multi-morbid population, where $35 \%$ have five or more chronic conditions(6).

Older age, being female or Hispanic, social isolation, smoking, having dementia, history of falls, use of walking assistive devices, and depression/anxiety are all associated with increased risk of becoming homebound(7)'(8)'(9). Longitudinal studies show conflicting data as to whether barriers at entry of the home were associated with becoming homebound(10)'(11). Other factors that are highly prevalent in the homebound population such as sensory impairment(12), pain(13)'(14), and sleep(13) have not been examined as potential risk factors for homebound progression. Current epidemiologic studies of homebound people do not distinguish those who are homebound due to physical vs cognitive impairment(7)' (15)' (16)' (17). Therefore, it is essential to differentiate between individuals with dementia and those with normal cognition who are homebound, to achieve a more personalized approach to their 
clinical care. The purpose of this study was to determine which potentially modifiable factors contribute to homebound progression (from independent living to needing assistance to homebound), stratified by dementia status.

\section{Methods}

\section{Study sample}

Data are from the original cohort of NHATS respondents in 2011 and the replenishment cohort in 2015. NHATS drew a random sample of individuals age 65 and older living in the contiguous US from Medicare enrollment files in 2010 (for the original cohort) and 2014 (for the replenishment cohort), with oversampling of those over 90 years old and nonHispanic blacks. We excluded nursing home respondents of NHATS in 2011 and in 2015 (baseline years for the original and replenishment cohorts). Interviews were completed annually and resulted in 11,528 unique respondents from both cohorts. Two-hour in-person interviews were conducted to collect self- or proxy-reported physical activity, functional status, chronic health conditions, and economic status. Physical and cognitive test batteries were conducted. Proxy answers consisted of $5.4 \%$ of all respondents at their baseline. Annual attrition from NHATS varied between $12.7 \%$ (between 2015 to 2016) to 18\% (2012-2013 and 2013-2014).

\section{Dementia categorization}

NHATS used three categories for cognitive status - probable dementia, possible dementia (or MCl), or cognitively normal(18) based on 3 factors: prior diagnosis by a physician through sampled person or proxy's self-reports, proxy scores on the AD8 Dementia Screening Interview (which includes temporal orientation, memory, judgment, and function tests), and additional in-person tests of memory, orientation, and executive function. Using the Aging, Demographics, and Memory Study (ADAMS) as a reference, the NHATS definition of dementia (probable and possible dementia combined) has a sensitivity of $\mathbf{8 5 . 7 \%}$ for dementia. Specificity is higher for the narrow definition (probable vs no dementia) (87.2\%)(18). We used the narrow definition of dementia (probable dementia vs no dementia) in this study, as we wanted those with dementia to be clearly differentiated from those without dementia in terms of their potentially modifiable risk factors. We dichotomized dementia based on the narrow definition and compared risk factors within those who had dementia or not. We did not track progression of cognitive impairment to dementia over time in this analysis.

\section{Homebound definition}

NHATS has no pre-defined measure of homebound status. We used Ornstein's definition of homebound status by creating frequency of respondents leaving home, whether respondent had difficulty leaving home, and whether help was required to leave home within the past month(1). Our categorizations of homebound status into 3 categories: independent, assist, and homebound, correspond to Ornstein's categorization of "not homebound", "semi-homebound", and "homebound.(1)"

\section{Primary outcome}

progression to homebound

We identified progression to homebound as an ordinal survival analysis with repeated measures of NHATS respondents followed annually over the 7 waves of NHATS from 2011 to 2017. Since respondents could change from assisted to independent or homebound in consecutive years or improve in their function over time, we compared the homebound classification for the last year available for each respondent vs their baseline year's homebound classification. Median follow-up years was 4, average 3.94 years, with minimum 1 year, maximum 7 years. Risks of 
institutionalization or death were included as possible outcomes in the progression definition. Most progression events did not include institutionalization or death $(2,485 / 3,330$ or $75 \%)$.

\section{Potential risk factors definitions}

All risk factors were assessed at baseline (either 2011 or 2015). We classified individuals as depressed if they responded that they lacked interest in usual activities or felt down or hopeless more than half of the days in the past month. We defined sleep problems needing over 30 min to fall asleep or having trouble falling back to sleep some nights, most nights, or every night. We defined social isolation in this study as having 2 or fewer persons in each respondent's social network since we adjusted for covariate of cohabitating with at least one person as marital status. Multimorbidity was defined as having at least 3 chronic conditions. All covariates (age, gender, ethnicity/race, education level, and marital status) were from baseline (either year 2011 or 2015 in the original or replenishment cohort, respectively).

\section{Data analysis}

We used Cox proportional hazard regression (SAS v. 9.4, Cary, NC) to analyze the risk of potentially modifiable variables on homebound progression. Model variables include adjusting for age, gender, race, marital status, and educational status. Potential modifiable predictor variables include environmental factors such as barrier at entry of home, home modifications, fear of falls or falls, depression, pain, poor sleep, multimorbidity, social isolation, and sensory (e.g. hearing or vision) impairments. Since there were multiple questions for certain variables, such as falls or hearing impairment, we chose the questions with $<10 \%$ of missing data. Among the modifiable variables, the variable environmental/home modification, which $35.9 \%$ respondents' answers were missing, and pain limiting function (72.5\% missing), and thus were excluded in the analysis.

\section{Results}


Table 1

Demographic factors related to homebound status and cognitive status at baseline, NHATS $2011 \& 2015(n=11,528)$.

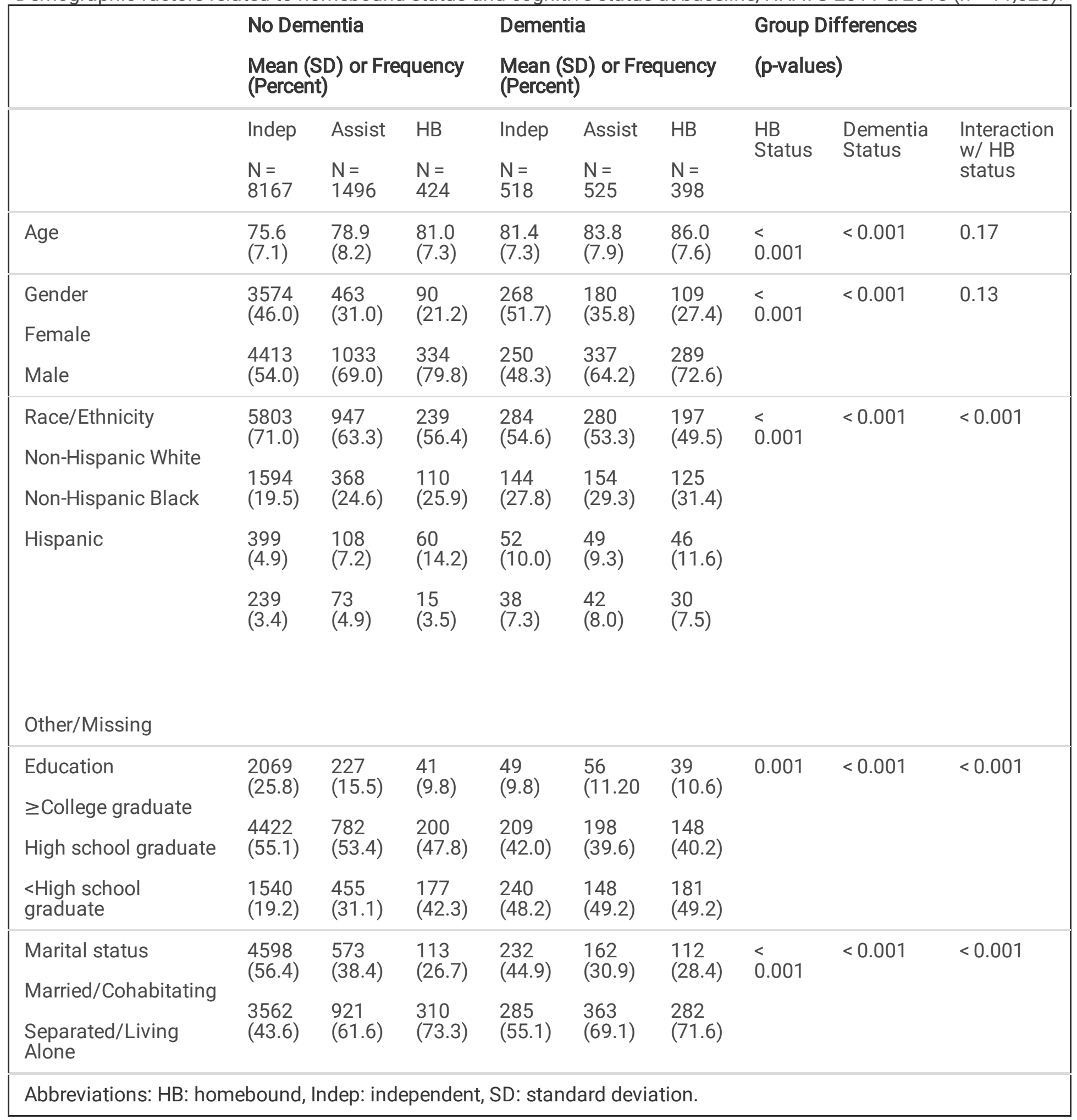

There were significant differences among those with dementia vs without dementia in age, gender, race/ethnicity, education, and marital status (Table 1). Those with dementia were more likely to be older, female, non-Hispanic Black, Hispanic, or other ethnicities. They were also more likely to have lower educational levels or live alone/separated. 
Table 2

Initial and final homebound status stratified by dementia status, NHATS 2011-2017 ( $\mathrm{n}=11,528)$.

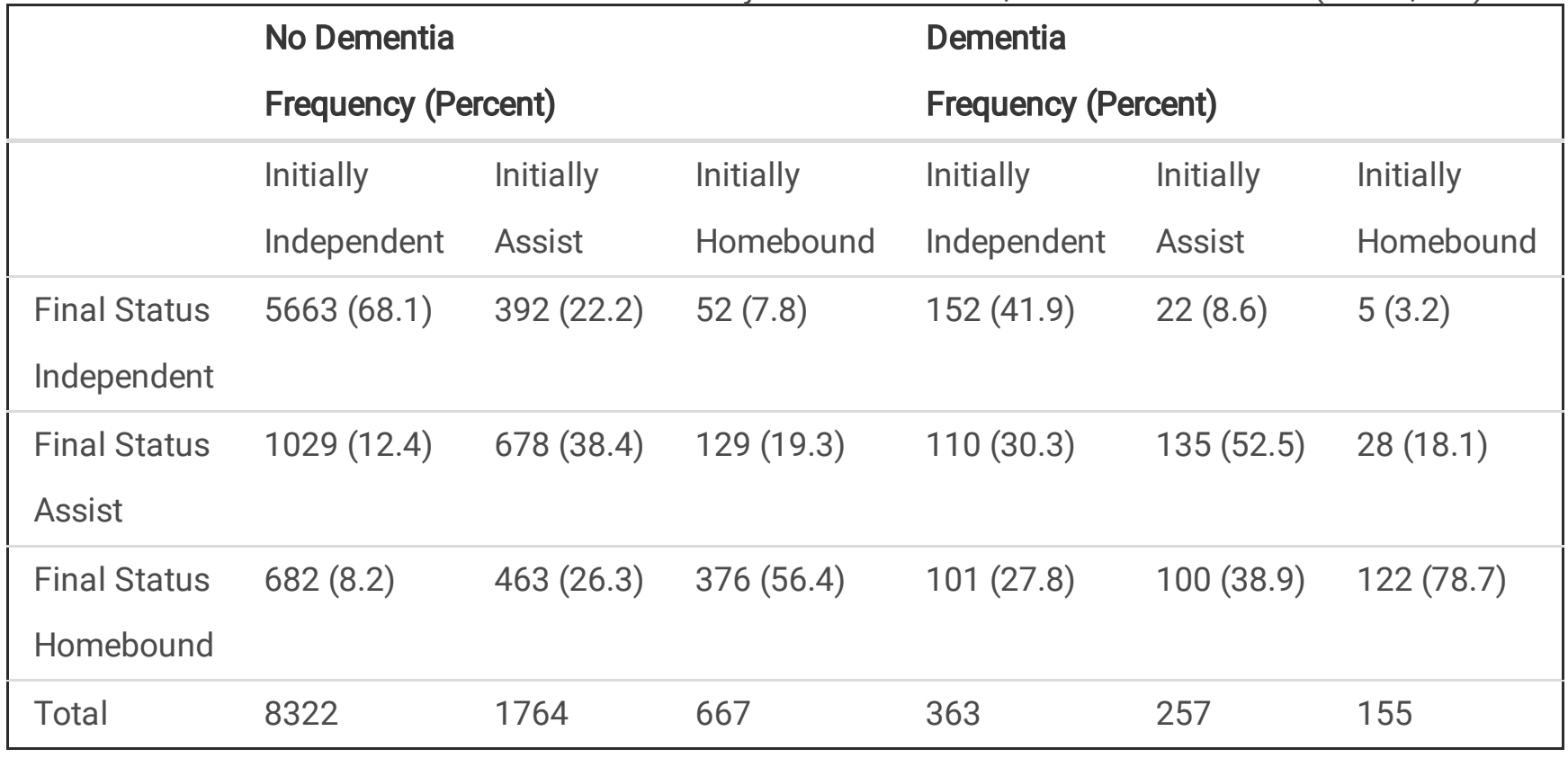

NHATS respondents with dementia had higher prevalence of being homebound compared to those without dementia, $27.8 \%$ vs $8.2 \%$ (Table 2). Persons with dementia were also more likely to remain homebound $(78.7 \%$ of those with dementia vs $56.4 \%$ of those without dementia). Persons with probable dementia were more likely to have progression events $(55 \%)$ than those with possible dementia (39.2\%) or those who were cognitively normal $(22.7 \%$, data not shown).

Table 3

Factors related to homebound progression based on proportional hazards regression among individuals living independently at baseline, with covariate adjustment for demographic factors, NHATS 2011-2017 ( $n=11,528)$. *95\% confidence interval excludes 1 .

\begin{tabular}{|llll|}
\hline Modifiable Factors & $\begin{array}{l}\text { No Dementia } \\
\text { HR }[95 \% \text { Cl] }\end{array}$ & $\begin{array}{l}\text { Dementia } \\
\text { HR }[95 \%]\end{array}$ & Interaction p-value \\
\hline Wears glasses & $1.080[0.994,1.173]$ & $1.052[0.91,1.217]$ & 0.85 \\
\hline Depression & $1.398[1.266,1.544]^{*}$ & $1.184[1.020,1.375] *$ & 0.11 \\
\hline Wears hearing aid & $0.938[0.841,1.047]$ & $0.910[0.743,1.116]$ & 0.31 \\
\hline Social isolation & $1.147[1.047,1.256]$ & $0.976[0.764,1.247]$ & 0.23 \\
\hline Stairs to enter home & $\mathbf{0 . 9 1 3}[0.836,0.998]^{*}$ & $1.011[0.867,1.179]$ & 0.06 \\
\hline Using cane/walker & $1.968[1.790,2.163]^{*}$ & $1.386[1.172,1.639] *$ & $<0.001$ \\
\hline Falls within past month & $1.382[1.229,1.555]^{*}$ & $1.334[1.126,1.579] *$ & 0.99 \\
\hline Worry about falls & $1.525[1.399,1.662]^{*}$ & $1.254[1.080,1.454]^{*}$ & 0.007 \\
\hline Bothered by pain & $1.198[1.104,1.300] *$ & $1.218[1.051,1.411] *$ & 0.99 \\
\hline Trouble sleeping & $1.070[0.986,1.162]$ & $0.977[0.843,1.133]$ & 0.34 \\
\hline
\end{tabular}

Depression, mobility impairments, and being bothered by pain are all potentially modifiable risk factors that significantly increased the risk of progression regardless of dementia status, after adjusting for age, gender, 
race/ethnicity, marital status, and educational status (Table 3). Depression increased the risk of homebound progression regardless of dementia status (interaction p-value 0.11). Mobility impairment (e.g., using a cane or walker) significantly increased risk of progression, regardless of dementia status (HR 1.97 for no dementia, HR 1.386 for dementia). However, use of assistive devices was more common among those with dementia (interaction p-value < 0.001). Both past history of falls or worry about falling were associated with increased risk of homebound progression, regardless of dementia status (HR 1.25-1.53). Neither wearing glasses or hearing aids posed a significant subsequent risk for progression regardless of dementia status. Social isolation only significantly affected the progression of homebound status among those without dementia (HR $1.147(1.047,1.256)$ ). Those who had stairs to enter the home had slightly reduced risk of progression, but only among those without dementia (HR $0.913(0.836,0.998))$.

\section{Discussion}

Among modifiable risk factors, depression, pain, and mobility impairments including falls increased the likelihood of homebound progression regardless of dementia status. Depression is present in $40 \%-60 \%$ of those who are homebound(9)'(1) and co-exists with dementia in about $20 \%$ of persons living with dementia(19). Screening for and treating depression is important as it is associated with increasing risk of homebound progression. Related to depression, social isolation was found to be associated with increased risk of homebound progression only among those without dementia. Signs of mobility limitation (e.g. using a cane or walker and recent falls) were strongly associated with an increased risk of homebound progression regardless of presence of dementia. Use of assistive devices among those without dementia was more highly associated with risk of homebound progression compared those with dementia, possibly because those with dementia become homebound primarily through cognitive rather than physical disability.

Sensory modifications such as glasses and hearing aids had no impact on homebound progression regardless of dementia subgroup, despite having a significant interaction with homebound status. This is in contrast to previous reports of self-reported vision and hearing impairment associated with shorter life expectancy and shorter duration of life without health problems(20). Homebound progression may be multifactorial; if so, examining sensory impairments alone may reveal no significant increased risk. Future studies can examine the risk of multiple factors as a cluster at predicting homebound progression.

Strengths of this study include using a nationally representative sample of community-dwelling older adults tracked longitudinally in terms of homebound progression. We also examined factors ranging from individual to environmental. The prospective data within NHATS allowed us to temporally examine baseline risk factors that precede the progression event. The dataset is generalizable and scaled to represent the entire older adults within the US. The identification of modifiable risk factors, depression, mobility, and pain, allow clinicians to inquire and treat these potentially reversible conditions to possibly prevent future functional decline. Policy makers could potentially use this information to advocate for long-term home-based support services, such as home health aides paid by Medicaid, to help older adults remain at home longer with slower progression to homebound status, institutionalization, and death. In addition, our study findings may be tested in interventions that specifically focus on these risk factors to see whether systematic identification and treatment may delay progression to becoming homebound.

This study has its limitations. Only $5.4 \%$ of those NHATS interviews were from proxy, yet $12.5 \%$ of NHATS respondents were classified as having dementia after objective cognitive testing. Among those homebound and had dementia, only $30 \%$ of responses were from proxies. It is possible that proxy answers for persons living with dementia may be inaccurate. For example, how often has the respondent had little interest or pleasure in doing things may be difficult for a proxy to distinguish from apathy of dementia. Another limitation is that only baseline risk factors were captured in 
the analysis, but many factors identified can vary over time. Further, many questions were focused on immobility over the past month, leading to reversals of homebound status over time. In addition, depending on when the NHATS interview was completed, responses may not reflect seasonal changes that may reduce ability to leave the home without assistance.

\section{Conclusion}

In conclusion, there are fewer than expected differences in risk factors for homebound progression between those with and without dementia. Depression, mobility problems, and pain are key risk factors to target in future intervention trials to evaluate whether treatment of these modifiable risk factors translate to preventing progression to homebound status.

\section{Declarations}

Ethics approval and consent to participate: National Health and Aging Trends Study was approved by Johns Hopkins University Institutional Review Board.

\section{Consent for publication: N/A}

Availability of data and materials: The datasets generated and analyzed during current study are publically available at www.nhats.org

Competing interests: all the authors have declared no competing interests.

\section{Funding: N/A}

Authors' contributions: MY conceived study concept, analyzed and interpreted data, wrote manuscript. NP analyzed data. ME analyzed and interpreted data. DE and JDW were major contributors in writing of the manuscript. All authors read and approved the final manuscript.

Acknowledgements: we express our appreciation to Karen Klein, of Clarus Editorial Services, for her edits and suggestions to the manuscript.

\section{References}

1. Ornstein KA, Leff B, Covinsky KE, Ritchie CS, Federman AD, Roberts L, et al. Epidemiology of the homebound population in the United States. JAMA Intern Med. 2015;175(7):1180-6.

2. Inc for OSORA CMS H. Medicare Benefit Policy Manual [Internet]. [cited 2018 Aug 21]. Available from: https://www.cms.gov/Regulations-and-Guidance/Guidance/Manuals/Downloads/bp102c07.pdf

3. Soones T, Federman AD, Leff B, Siu AL, Ornstein KA. Two-year mortality among homebound, older adults: an analysis of the National Health and Aging Trends Study. J Am Geriatr Soc. 2017;65(1):123-9.

4. Alzheimer's Facts and Figures Report | Alzheimer's Association [Internet]. [cited 2018 Oct 28]. Available from: https://alz.org/alzheimers-dementia/facts-figures

5. Bynum JPW, Rabins P V., Weller W, Niefeld M, Anderson GF, Wu AW. The relationship between a dementia diagnosis, chronic illness, medicare expenditures, and hospital use. Res Pract Alzheimers Dis. 2005;10:160-4.

6. Mondor L, Maxwell CJ, Hogan DB, Bronskill SE, Gruneir A, Lane NE, et al. Multimorbidity and healthcare utilization among home care clients with dementia in Ontario, Canada: A retrospective analysis of a population-based cohort. 
PLoS Med. 2017;14(3):1-17.

7. Xiang X, Chen J, Kim M. Trajectories of Homebound Status in Medicare Beneficiaries Aged 65 and Older. Gerontologist [Internet]. 2019 Mar 13 [cited 2019 Apr 4]; Available from:

https://academic.oup.com/gerontologist/advance-article/doi/10.1093/geront/gnz023/5377640

8. Katsumata Y, Arai A, Tamashiro H. Contribution of falling and being homebound status to subsequent functional changes among the Japanese elderly living in a community. Arch Gerontol Geriatr. 2007;45(1):9-18.

9. Xiang, Xiaoling, Brooks J, Xiang X, Brooks J. Correlates of Depressive Symptoms among Homebound and SemiHomebound Older Adults. J Gerontol Soc Work. 2017 Apr;60(3):201-14.

10. Smith AR, Chen C, Clarke P, Gallagher NA. Trajectories of Outdoor Mobility in Vulnerable Community-Dwelling Elderly: The Role of Individual and Environmental Factors. J Aging Health. 2016;28(5):796-811.

11. Cohen-Mansfield J, Shmotkin D, Hazan H. The effect of homebound status on older persons. J Am Geriatr Soc. 2010 Dec;58(12):2358-62.

12. Wang J, Kearney JA, Jia H, Shang J. Mental Health Disorders in Elderly People Receiving Home Care: Prevalence and Correlates in the National U.S. Population. Nurs Res. 2016;65(2):107-16.

13. Patel K V., Guralnik JM, Phelan EA, Gell NM, Wallace RB, Sullivan MD, et al. Symptom Burden Among CommunityDwelling Older Adults in the United States. J Am Geriatr Soc. 2019;67(2):223-31.

14. Wajnberg A, Ornstein K, Zhang M, Smith KL, Soriano T. Symptom Burden in Chronically III Homebound Individuals HHS Public Access. J Am Geriatr Soc [Internet]. 2013 [cited 2019 Apr 22];61(1):126-31. Available from: https://www-ncbi-nlm-nih-gov.go.libproxy.wakehealth.edu/pmc/articles/PMC4784248/pdf/nihms764681.pdf

15. Ornstein KA, Garrido MM, Bollens-Lund E, Reckrey JM, Husain M, Ferreira KB, et al. The Association Between Income and Incident Homebound Status Among Older Medicare Beneficiaries. J Am Geriatr Soc [Internet]. 2020 Nov 1 [cited 2021 Feb 1];68(11):2594-601. Available from: https://pubmed.ncbi.nlm.nih.gov/32776512/

16. De-Rosende Celeiro I, Santos-del-Riego S, Muñiz García J, De-Rosende Celeriro I. Homebound status among middleaged and older adults with disabilities in ADLs and its associations with clinical, functional, and environmental factors. Disabil Health J [Internet]. 2017 [cited 2018 Nov 25];10(1):145-51. Available from: https://wwwclinicalkey-com.go.libproxy.wakehealth.edu/\#!/content/playContent/1-s2.0-S1936657416300942? returnurl=null\&referrer=null

17. Zhao Y, Alderden J, Lind B, Stibrany J. Risk factors for falls in homebound community-dwelling older adults. Public Health Nurs [Internet]. 2019 Nov 1 [cited 2021 Feb 4];36(6):772-8. Available from:

https://pubmed.ncbi.nlm.nih.gov/31407384/

18. Kasper JD, Freedman VA, Spillman B. Classification of Persons by Dementia Status in the National Health and Aging Trends Study. 2013;14. Available from: www.NHATS.org.

19. Lyketsos CG, Lopez O, Jones B, Fitzpatrick AL, Breitner J, DeKosky S. Prevalence of Neuropsychiatric Symptoms in Dementia and Mild Cognitive Impairment. JAMA [Internet]. 2002 Sep 25 [cited 2019 Aug 27];288(12):1475. Available from: http://jama.jamanetwork.com/article.aspx?doi=10.1001/jama.288.12.1475

20. Tareque MI, Chan A, Saito Y, Ma S, Malhotra R. The Impact of Self-Reported Vision and Hearing Impairment on Health Expectancy. J Am Geriatr Soc. 2019 Dec 1;67(12):2528-36.

21. Penninkilampi R, Casey AN, Singh MF, Brodaty H. The Association between Social Engagement, Loneliness, and Risk of Dementia: A Systematic Review and Meta-Analysis. J Alzheimer's Dis. 2018;66(4):1619-33. 